\title{
Effects of Geniposide and Geniposidic Acid on Fluoxetine-Induced Muscle Atrophy in C2C12 Cells
}

\author{
Shang-Ming Huang ${ }^{1}$, Shuan-Ying Lin ${ }^{1}$, Ming-Kai Chen ${ }^{1}$, Chiung-Chi Peng ${ }^{2}$ and Chiu-Lan Hsieh ${ }^{1, *}$ \\ 1 Department of Biology, National Changhua University of Education, 1 Jin-De Rd., Changhua 50007, Taiwan; \\ zxzxmj2323@hotmail.com (S.-M.H.); aas530504@gmail.com (S.-Y.L.); zse191@gmail.com (M.-K.C.) \\ 2 Graduate Institute of Clinical Medicine, Taipei Medical University, 250 Wu-Xing St., Taipei 11031, Taiwan; \\ misspeng@tmu.edu.tw \\ * Correspondence: clhsieh@cc.ncue.edu.tw; Tel.: +886-4-7232105 (ext. 3461); Fax: +886-4-7211156
}

check for updates

Citation: Huang, S.-M.; Lin, S.-Y.; Chen, M.-K.; Peng, C.-C.; Hsieh, C.-L. Effects of Geniposide and Geniposidic Acid on FluoxetineInduced Muscle Atrophy in C2C12 Cells. Processes 2021, 9, 1649. https://doi.org/10.3390/pr9091649

Academic Editor: Jer-An Lin

Received: 20 August 2021

Accepted: 10 September 2021

Published: 13 September 2021

Publisher's Note: MDPI stays neutral with regard to jurisdictional claims in published maps and institutional affiliations.

Copyright: (c) 2021 by the authors. Licensee MDPI, Basel, Switzerland. This article is an open access article distributed under the terms and conditions of the Creative Commons Attribution (CC BY) license (https:/ / creativecommons.org/licenses/by/ $4.0 /)$.
Abstract: Fluoxetine, an antidepressant known as a selective 5-hydroxytryptamine reuptake inhibitor (SSRI), can cause side effects such as muscle atrophy with long-term use, but the mechanism is not fully understood. Geniposide (GPS) and geniposidic acid (GPSA), the main components of Gardenia jasminoides fruit, have been shown to have biological activity in disease prevention, but their role in preventing FXT-related side effects such as muscle atrophy remains unclear. The process of muscle atrophy is a complex physiological mechanism involving the balance of protein synthesis and catabolism. In this study, we hypothesized that FXT may suppress hypertrophy signaling and activate the atrophy mechanisms, resulting in proteolysis and reduced protein synthesis, while geniposide (GPS) and geniposide acid (GPSA) may be beneficial in improving muscle weakness caused by FXT. The C2C12 cell model was used to examine the expression of hypertrophy signaling (PI3K, Akt, and mTOR) and protein break signals (FOXO, MuRF-1, and MyHC). Our data indicated that FXT inhibited MyHC and promoted MuRF-1 protein expression by downregulating the signaling pathways of p-ERK1/2, p-Akt, p-mTOR, and p-FOXO, resulting in a decrease in differentiation and myotube formation in $\mathrm{C} 2 \mathrm{C} 12$ muscle cells, which further resulted in muscle atrophy. However, GPS and GPSA can positively regulate the atrophy mechanism induced by FXT in muscle cells, thereby ameliorating the imbalance in muscle synthesis. In conclusion, GPS and GPSA have the potential to attenuate the muscle loss caused by long-term FXT administration, diseases, or the aging process.

Keywords: fluoxetine; geniposide; geniposidic acid; muscle atrophy

\section{Introduction}

With the increased stress of modern life, the number of people suffering from psychiatric disorders is also increasing. Fluoxetine (FXT), such as Prozac, is the typical clinical antidepressant known as a selective 5-hydroxytryptamine reuptake inhibitor (SSRI) used to treat depression, bulimia, obsessive-compulsive disorder (OCD), panic disorder, and premenstrual dysphoric disorder (PMDD) [1]. However, SSRI drugs also have a considerable number of side effects including nausea, upset stomach, constipation, headache, anxiety, insomnia, drowsiness, dizziness, nervousness, palpitations, abnormal appetite, weight changes, cold symptoms (stuffy nose, sneezing, and sore throat), dry mouth, decreased sexual function, etc. [1,2]. Recently, muscle atrophy caused by SSRI drugs has received increasing attention. The literature mentions that a large number of patients have developed muscle atrophy after ingesting SSRI drugs, especially when the patient taking Prozac is female, older than 60 years old, and has been taking the drug for about a month [3].

Muscle atrophy is a complex set of physiological mechanisms involving an imbalance in muscle protein synthesis and catabolism [4]. Briefly, muscle atrophy can be described as a decrease in muscle protein synthesis and an increase in muscle protein catabolism, resulting in a net negative balance of muscle proteins [5]. The degradation of skeletal muscle proteins 
is controlled by two protein hydrolysis pathways, including the ubiquitin-proteasome system and autophagy [6,7]. Previously, the FOXO (mammalian forkhead members of the class O) transcription factor has been identified as the main coordinator of protein hydrolysis pathways, which can induce the expression of genes associated with muscle metabolic mechanisms, such as several autophagy-related genes as well as the ubiquitin ligases Atrogin-1 and MuRF-1 [8-10]. FOXO family proteins have important roles in metabolism, cell proliferation, anti-stress, and apoptosis and are involved in many pathological and physiological processes including autophagy, metabolism, inflammation, cytokine expression, immunity, differentiation, and resistance to oxidative stress [11]. FoxO1 and FoxO3 are key factors in muscle energy homeostasis by controlling glycolytic and lipolytic fluxes as well as mitochondrial metabolism [11,12]. They are also key regulators of proteolysis, as they regulate the activities of several players in the ubiquitin-proteasome and autophagylysosome proteolytic pathways, including mitochondrial autophagy, which is also known as mitophagy $[13,14]$.

The fruits of Gardenia jasminoides Ellis (Rubiaceae) have been widely adopted in traditional medicine formulations for the treatment of diseases [15]. The pharmacological effects of gardenia have been reported to be hypoglycemic, lipid-regulating, neuroprotective, anti-inflammatory, antioxidant, and antithrombotic, with geniposide (GPS) being the main active ingredient $[16,17]$. GPS is a natural product of iridoid glycosides that can be isolated from gardenia fruits and has now been shown to possess a wide range of effects, including antioxidant, anti-inflammatory, and antidiabetic activities [16]. By contrast, geniposidic acid (GPSA), one of the components of gardenia with structural similarity to GPS, is still poorly reported in the literature.

Overall, many side effects of FXT have been documented, but muscle weakness is one of the most overlooked side effects, and the long-term use of fluoxetine may result in muscle loss. In our preliminary study, FXT was found to cause high mortality and teratogenicity in $\mathrm{HH}$ stage 10 chicken embryos with severe necrosis, edema, and muscle fragmentation on tissue sections. In addition, the protective effect of GPS and GPSA on muscle atrophy caused by FXT remains unclear, although gardenia components such as GPS and GPSA have been shown to have biological benefits in disease prevention.

This study aimed to investigate the effects of FXT on muscle atrophy and muscle protein metabolism in $\mathrm{C} 2 \mathrm{C} 12$ muscle cells and to investigate the protective mechanisms of GPS and GPSA against FXT-induced muscle cell damage. We hypothesized that FXT could inhibit hypertrophic signaling and activate atrophic signaling, resulting in reduced proteolysis and protein synthesis, while GPSA and GPS may be beneficial in reducing FXT-induced muscle weakness.

\section{Materials and Methods}

\subsection{Chemicals}

Geniposide ( $\geq 98 \%$, HPLC), geniposidic acid ( $\geq 98 \%$, HPLC), Tri-reagent, fluoxetine hydrochloride, bovine serum albumin (BSA), sodium dodecyl sulfate (SDS), hematoxylin, and Eosin Y were purchased from Sigma-Aldrich (St. Louis, MI, USA). Thirty percent acrylamide/bis and Bio-Rad Protein assay were products of Bio-Rad Laboratories (Hercules, CA, USA). Diethylpyrocarbonate (DEPC)-treated water was supplied by BioBasic Inc. (Markham, Canada). HiScript $\mathrm{I}^{\mathrm{TM}}$ First Strand cDNA Synthesis Kit was purchased from Bionovas Co. (Washington, DC, USA). A 2X PCR Master Mix (Red) was supplied by Faith Co. (Taipei, Taiwan). Dulbecco's Modified Eagle Medium (DMEM) powder and $0.05 \%$ trypsin-EDTA (1X) (TE) were products of Gibco Co. (Grand Island, NE, USA). The 100X Penicillin streptomycin solution was a product of Corning Co. (Corning, NY, USA). Nuclear/Cytosol Fractionation Kit was provided by BioVision Co. (Milpitas, CA, USA). RIPA buffer was a product of Genestar Co. (Houston, TX, USA). Immobilon Western Chemiluminescent HRP Substrate and PVDF transfer membrane were supplied by Millipore Co. (Burlington, MA, USA). Geniposide and geniposidic acid were provided by Lin-Chuan-Zi-Shin Biotech Co. (China). Antibodies of total Akt, mTOR and FOXO1, 
and phosphorylated proteins were purchased from Cell Signaling Co. (Danvers, MA, USA). Antibodies of TRIM63 (MuRF1) and FBXO32 (Atrogin-1 or MAFbx) were supplied by Protein Tech (Rosemont, IL, USA). The antibody of $\beta$-actin was provided by Thermo Co. (Waltham, MA, USA), and the antibody of MF20 (myosin heavy chain, MyHC) was purchased from DSHB Co. (Iowa City, IN, USA).

\subsection{Cell Culture and Differentiation}

C2C12 cell line, a mouse muscle myoblast, was obtained from the Bioresource Collection and Research Center (BCRC, Food Industry Research and Development Institute, Hsin Chu, Taiwan). Cells were grown in a DMEM medium supplemented with $10 \%$ FBS, $4.5 \mathrm{~g} / \mathrm{L}$ glucose, $4 \mathrm{mM}$ L-glutamine, $1.5 \mathrm{~g} / \mathrm{L}$ sodium bicarbonate, and 1\% PS antibiotic solution (100 unit $/ \mathrm{mL}$ ) at $37^{\circ} \mathrm{C}$ in a humidified atmosphere of $95 \%$ air and $5 \% \mathrm{CO}_{2}$. When the cell density reached approximately $80 \%, 0.05 \%$ trypsin-EDTA (TE) was added to digest the cells for subculture [18]. For differentiation, subcultured cell populations were counted, seeded into 6-well plates and 10-cm flat bottomed plates, and incubated at $37^{\circ} \mathrm{C}$ and $5 \% \mathrm{CO}_{2}$ atmosphere for $72 \mathrm{~h}$ until confluence. The medium was sucked away, the cells were washed once with serum-free medium, and cell fusion was induced in a medium containing $2 \%$ horse serum (differentiation medium). The fusion status was documented at 3,5 , and 7 days after switching to the differentiation medium.

\subsection{Cell Viability Assay}

Cell viability was determined by using the MTT assay and modified according to the method in the literature [19]. C2C12 cells were seeded at a density of $8 \times 10^{4}$ cells $/ \mathrm{mL}$ onto 6-well plates and cultured as described above for 4-6 days in order to promote differentiation. Next, different indicated concentrations of fluoxetine (dissolved in distilled water at a concentration of 5-20 $\mu \mathrm{M}$ ), geniposide (dissolved in distilled water at a concentration of 50-400 $\mu \mathrm{M})$, and geniposidic acid (50-400 $\mu \mathrm{M}$ dissolved DMSO at a concentration of $50-400 \mu \mathrm{M})$ were added to the cells, respectively, and the incubation continued for 48 $\mathrm{h}$. The used medium was removed, the cells are rinsed twice with PBS, $1 \mathrm{~mL}$ of $0.5 \%$ MTT solution was added (50 mg MTT powder dissolved in $10 \mathrm{~mL}$ PBS), and incubation continued at $37^{\circ} \mathrm{C}$ for $2 \mathrm{~h}$. The MTT solution was removed and $0.5 \mathrm{~mL}$ of fresh DMSO was added and left in the dark for $30 \mathrm{~min}$. The DMSO solution aspirated, and its optical density was read at $570 \mathrm{~nm}$ using an ELISA reader (Thermo Fisher Scientific Inc., Waltham, MA, USA). Cell viability was calculated according to Equation (1).

$$
\text { Cell viability }(\%)=\left(A_{570 \mathrm{~nm}, \text { sample }} / A_{570 \mathrm{~nm}, \text { control }}\right) \times 100 \% \text {. }
$$

\subsection{Analysis of Muscle Atrophy-Related Protein Expressions by Western Blot}

The cytosolic proteins were isolated from $\mathrm{C} 2 \mathrm{C} 12$ muscle cells after cell differentiation and treatment with the drugs. Total protein was extracted in $500 \mathrm{~L}$ of lysis buffer containing $150 \mathrm{mM} \mathrm{NaCl}, 1.0 \% \mathrm{NP}-40,0.5 \%$ sodium deoxycholate, $0.1 \%$ SDS, $50 \mathrm{~mL}$ pH 7.5 Tris, $1 \mathrm{mM}$ PMSF, and $10 \mathrm{mg} / \mathrm{mL}$ leupeptin. The reaction mixture was placed on ice for $5 \mathrm{~min}$, and the cells were then scraped off. The collected cell pellets were ultracentrifuged at 12,000 rpm for $15 \mathrm{~min}$ at $4{ }^{\circ} \mathrm{C}$. The cytosolic fraction (supernatant) proteins were measured by Bradford assay using BSA as the standard. The total cytosolic extracts were separated on $10 \%$ SDSpolyacrylamide minigels for target protein detection, including MyHC (250 kDa), MuRF-1 (40 kDa), Atrogin-1 (50 kDa), phosphorylated FOXO1 (Ser256, $82 \mathrm{kDa})$, phosphorylated Akt (Ser473, $60 \mathrm{kDa}$ ), phosphorylated mTOR (Ser2481, $289 \mathrm{kDa}$ ), and phosphorylated ERK (Thr202/Tyr204, 42/44 kDa), and then transferred to Immobilon polyvinylidene difluoride membrane (PVDF; Millipore, Bedford, MA, USA) with the transfer buffer consisting of $25 \mathrm{mM}$ Tris- $\mathrm{HCl}$ (pH 8.9), $192 \mathrm{mM}$ glycine, and 20\% methanol. The membrane was blocked with $5 \%$ BSA solution for $1 \mathrm{~h}$ at room temperature and then incubated overnight at $4{ }^{\circ} \mathrm{C}$ with the indicated primary antibodies (1:1000 dilutions). After hybridization with the primary antibody, the PVDF membranes were washed three times with Tris-buffered saline 
Tween-20 (TBST). The PVDF membranes were then immersed in chemiluminescent HRP substrate (Millipore, Bedford, MA, USA) for approximately $15 \mathrm{~s}$ for visualization and analysis. The protein density of the cells was quantified using Image J software (National Institutes of Health, Bethesda, MD, USA).

\subsection{Counting of Myotubes by Hematoxylin-Eosin Staining}

Myotubes from C2C12 myoblasts were counted by hematoxylin-eosin staining as described by Joshi et al. (2011) [19]. After differentiation and drug treatment, C2C12 cells were rinsed twice with PBS, fixed with formaldehyde for $10 \mathrm{~min}$, and then rinsed once with PBS, followed by dehydration with $100 \%, 95 \%, 80 \%$, and $75 \%$ ethanol for $1 \mathrm{~min}$. Cells were rinsed once more with DDI water, then stained with hematoxylin for 5 min and treated with $70 \%$ ethanol containing $0.5 \%$ hydrochloric acid to remove cytoplasmic color, followed by alkalization with $1 \% \mathrm{NaHCO}_{3}$. After removing the residual alkaline solution with DDI water, the cells were stained with Eosin Y for $1 \mathrm{~min}$. Then, the excess dye was removed by gradient exposure with $75 \%, 80 \%, 95 \%$, and 100\% ethanol for $1 \mathrm{~min}$. Micrographs of the cells were taken at 200X magnification in three different randomly selected areas. Image J was used to count the total number of myotubes. At least one hundred cells should be counted, and the total number of myotubes was statistically processed and graphed.

\subsection{Statistical Analysis}

The data obtained in the experiments were statistically processed using the Statistical Package for the Social Science (SPSS) version 15.0. Data were expressed as mean \pm SD. Statistical comparisons were performed by one-way analysis of variance (ANOVA), followed by Duncan's multiple comparison test. The differences are considered significant when the $p$-value is $<0.5$.

\section{Results and Discussion}

3.1. Effects of GPS and GPSA on FXT-Inhibited Differentiation and Myotube Formation in C2C12 Muscle Cells

C2C12 muscle cells with or without FXT treatment are shown in Figure 1. Under phase-contrast microscopy, cells that have not been treated with FXT appear smooth and orderly (Figure 1a). Although FXT did not show any inhibitory effect on cell viability in the dose range of $5 \mu \mathrm{M}-20 \mu \mathrm{M}$ (Figure 1b), the number of myotubes decreased in a dosedependent manner in response to FXT (Figure 1a). Similarly, GPS and GPSA did not show any inhibition of cell viability in the dose range of 50 to $400 \mu \mathrm{M}$ (Figure 1c,d), indicating that the reduction of myotubes formed by C2C12 cell differentiation was not associated with any cytotoxic effect of FXT, GPS, and GPSA. The differentiation of C2C12 cells required at least 4 days (Figure 2a). Treatment with GPS (50 $\mu \mathrm{M}$ and $400 \mu \mathrm{M}$, respectively) and/or GPSA (50 $\mu \mathrm{M}$ and $400 \mu \mathrm{M}$, respectively) effectively attenuated the inhibitory effect of FXT $(20 \mu \mathrm{M})$ on C2C12 cell differentiation (Figure $2 \mathrm{~b})(p<0.05)$. In addition, as shown in Figure 3, H\&E staining (Figure 3a) showed that both GPS and GPSA could increase the number of myotubes inhibited by FXT at $50 \mu \mathrm{M}$ and $400 \mu \mathrm{M}$, respectively, and the quantitative histograms clearly show the change in the number of myotubes (Figure $3 b$ ). 

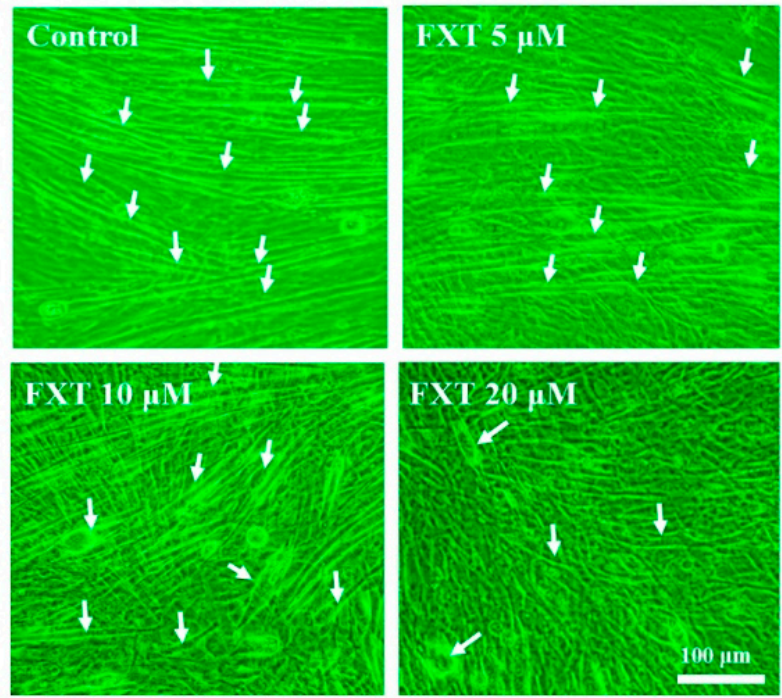

(a)

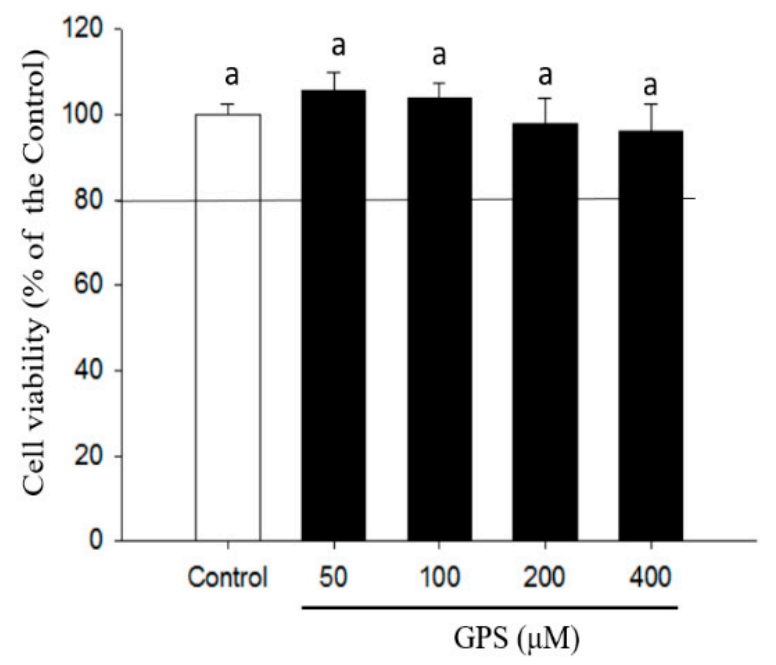

(c)

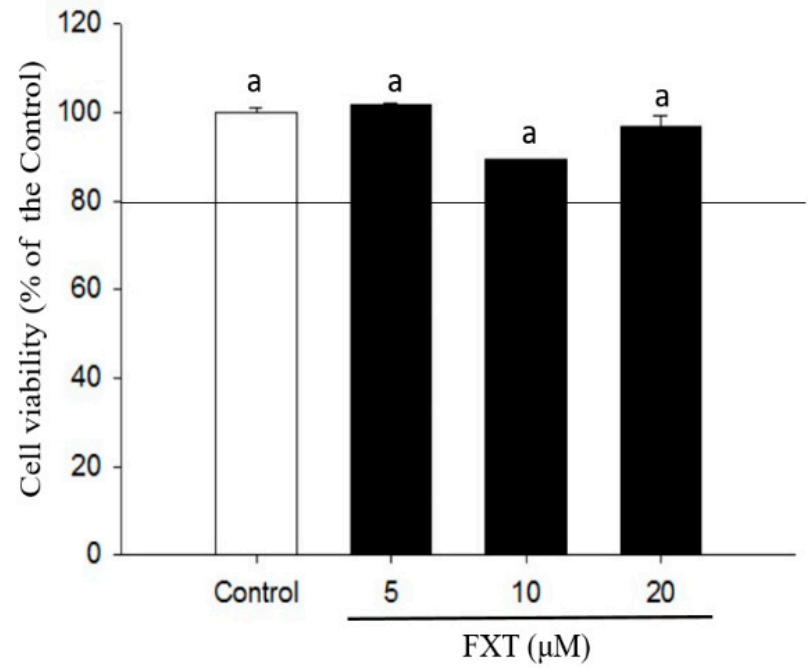

(b)

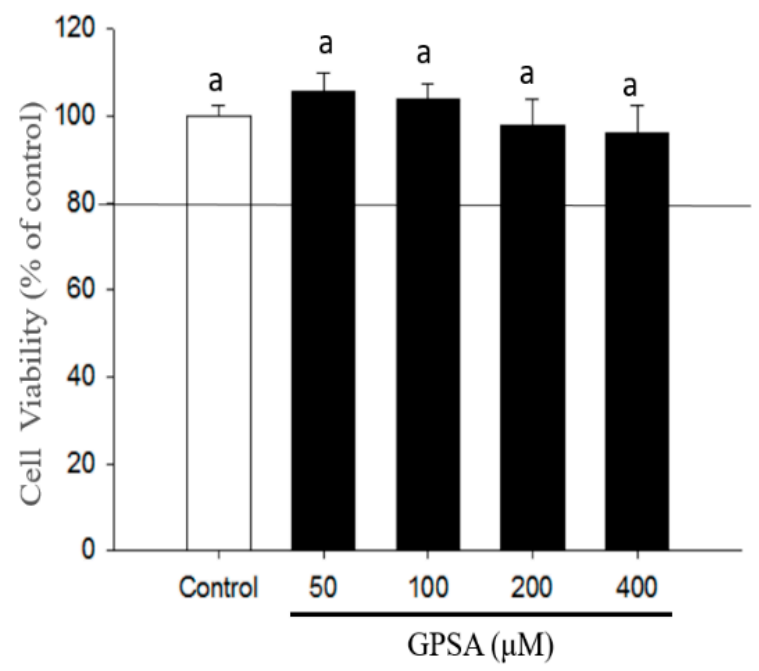

(d)

Figure 1. Myotube morphology and cell viability of C2C12 muscle cells treated or untreated with FXT, GPS, or GPSA. (a) $\mathrm{C} 2 \mathrm{C} 12$ muscle cells treated with 5-20 $\mu \mathrm{M}$ FXT for $48 \mathrm{~h}$, compared with the control group without FXT treatment. Myotubes were examined with a phase-contrast light microscope (Olympus CKX41). Original magnification, $\times 200$. Scale bar indicates $100 \mu \mathrm{m}$. The arrow signs indicate the survival myotubes. Cell viability was affected by (b) FXT (5-20 $\mu \mathrm{M})$, (c) GPS (50-400 $\mu \mathrm{M})$, and (d) GPSA (50-400 $\mathrm{MM})$. C2C12 muscle cells were differentiated after $48 \mathrm{~h}$ of culture for 4 days. Data are expressed as mean $\pm \operatorname{SD}(n=3)$. Different letters in lowercase indicate significance between one another as $p<0.05$. 


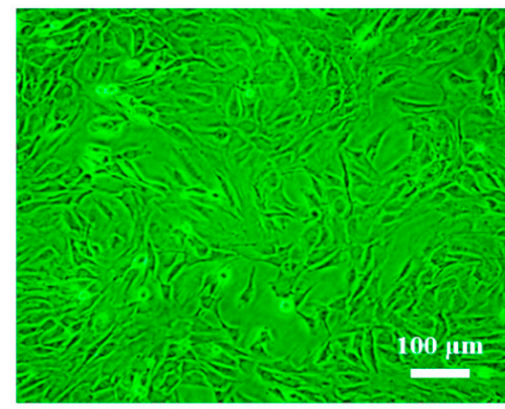

Day 0

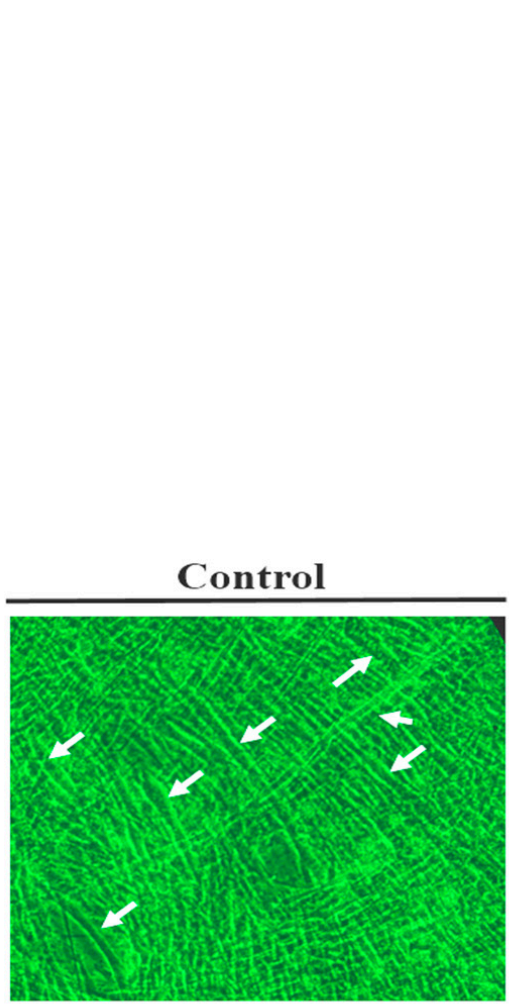

FXT

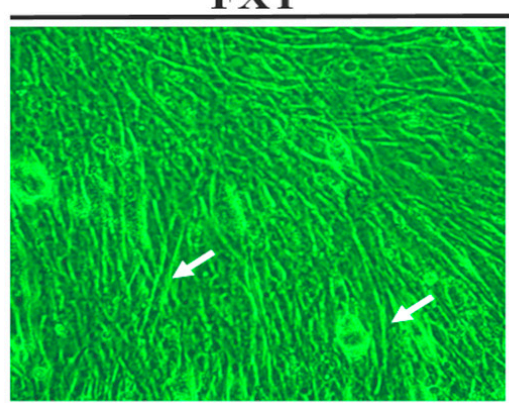

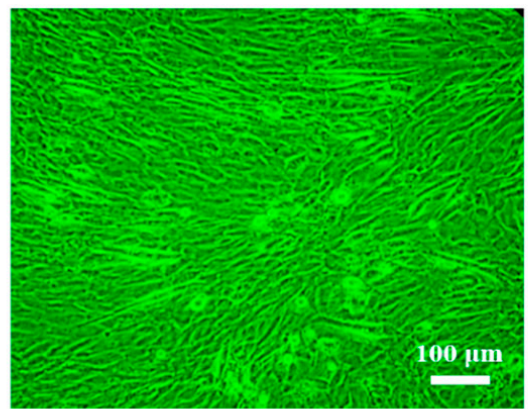

Day 1

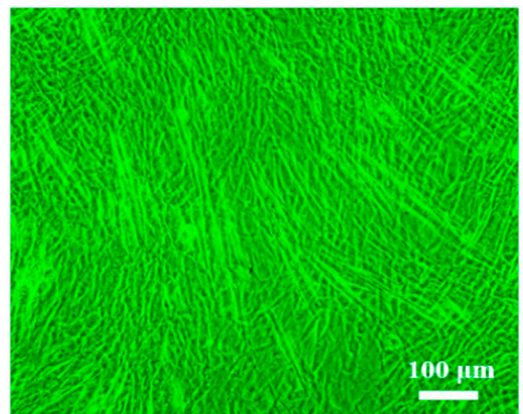

Day 3

(a)

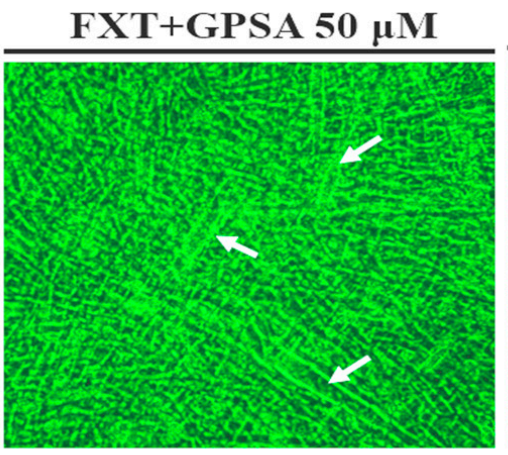

FXT+GPS $50 \mu M$

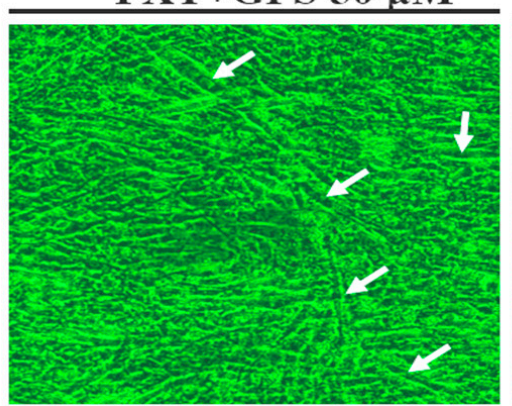

(b)

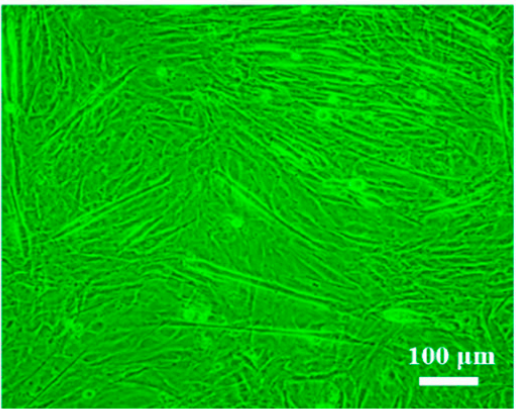

Day 2

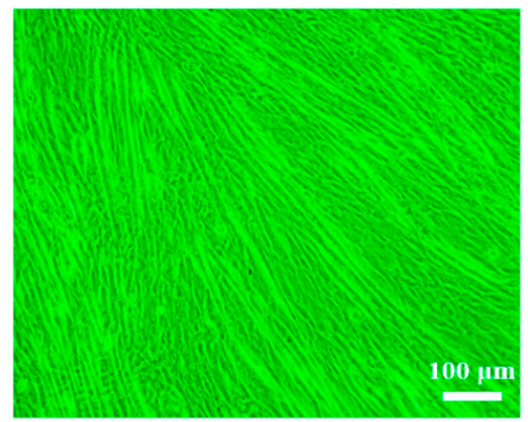

Day 4

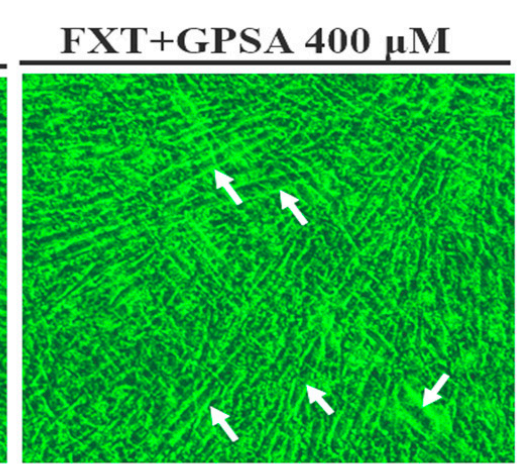

FXT+GPS $400 \mu \mathrm{M}$

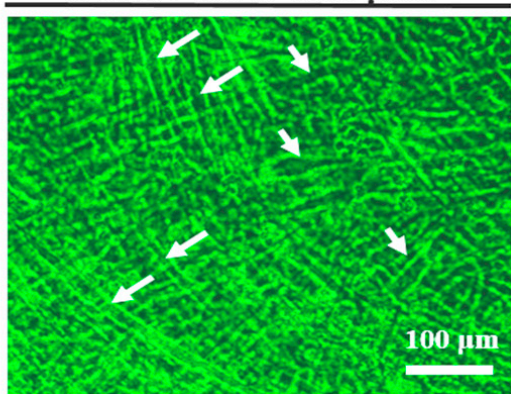

00 inim

Figure 2. Effect of GPSA and GPS on the differentiation of FXT-treated C2C12 muscle cells. (a) Phase-contrast images of the undifferentiated (day 0) and the differentiated (day 1-day 4) C2C12 muscle cells. (b) C2C12 cells were differentiated in 6-well plates for 4 days and then treated with PBS (vehicle control), FXT (20 $\mu \mathrm{M})$, GPSA (50 $\mu \mathrm{M}$ and $400 \mu \mathrm{M})$, and GPS $(50 \mu \mathrm{M}$ and $400 \mu \mathrm{M})$ for $48 \mathrm{~h}$, respectively. Myotubes were examined with a phase-contrast light microscope (Olympus CKX41). Original magnification, $\times 200$. Scale bar indicates $100 \mu \mathrm{m}$. The arrow signs indicate the survival myotubes. 


\section{Control}

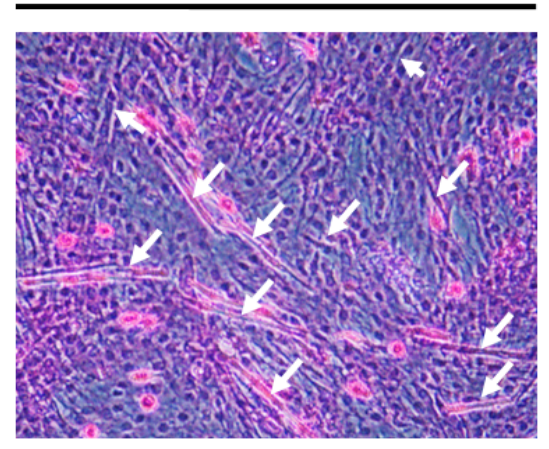

FXT

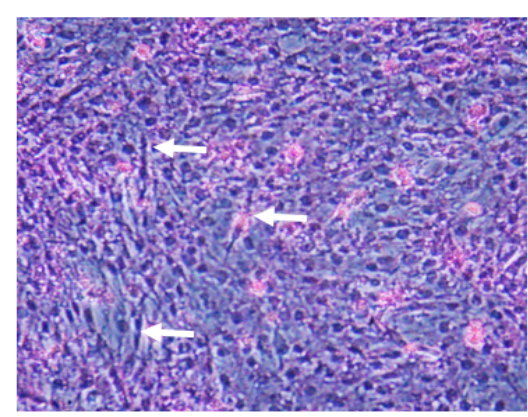

FXT+GPSA $50 \mu M$

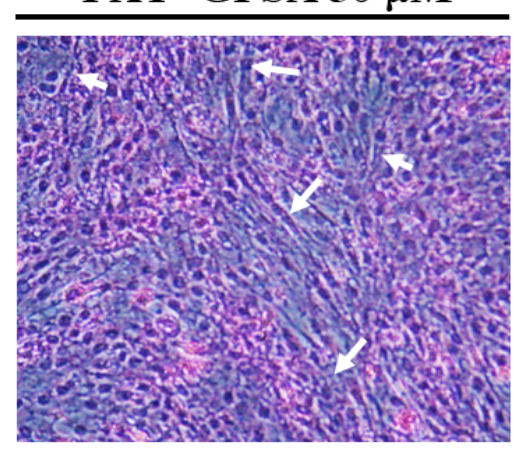

FXT+GPS $50 \mu M$

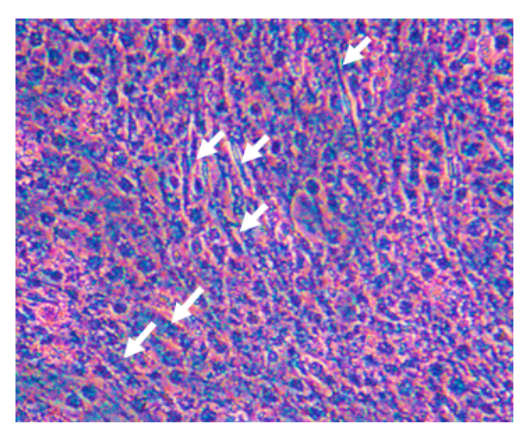

\section{FXT+GPSA $400 \mu M$}

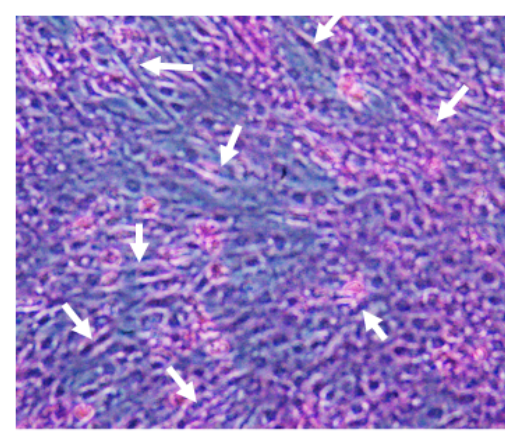

FXT+GPS $400 \mu M$

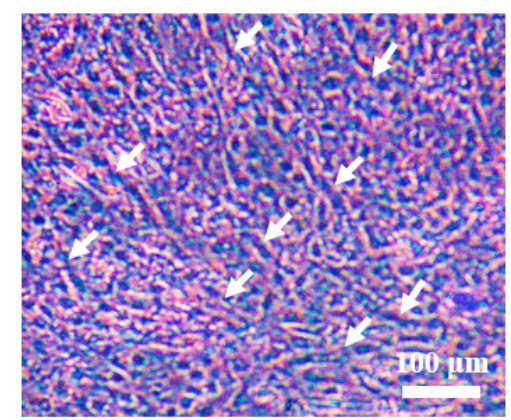

(a)

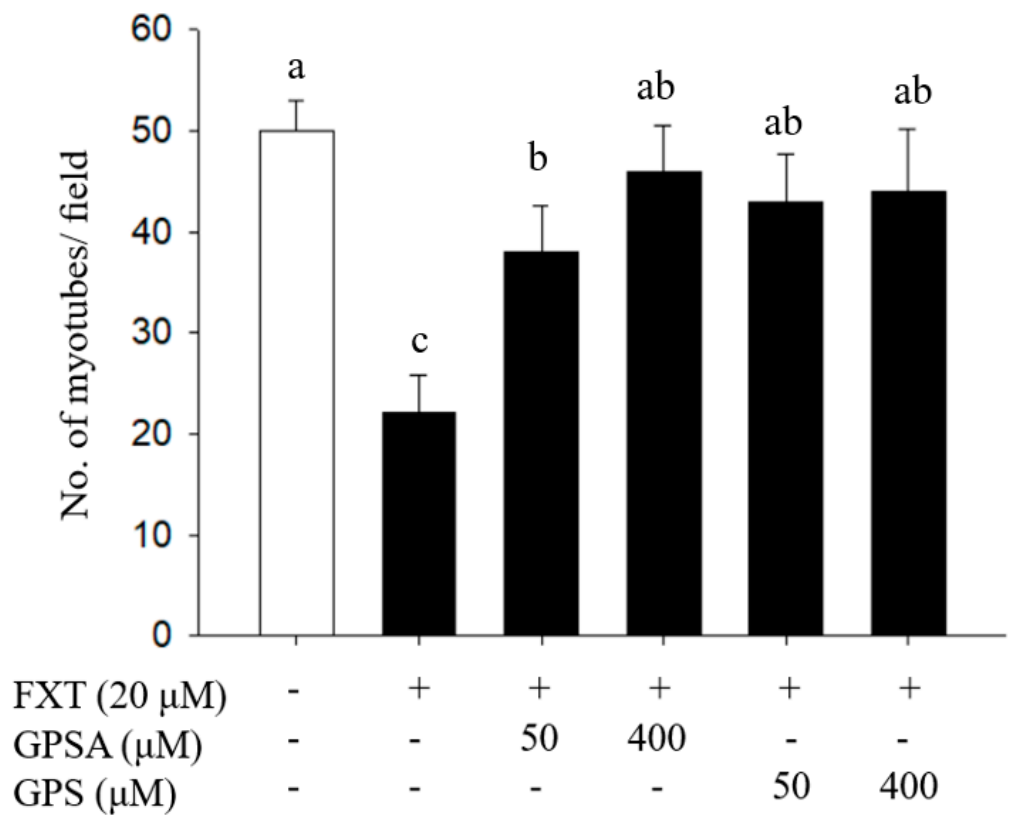

(b)

Figure 3. Effect of GPSA and GPS on myotube formation in FXT-treated C2C12 muscle cells. C2C12 cells were treated with PBS (vehicle control), FXT $(20 \mu \mathrm{M})$ together with GPSA (50 and $400 \mu \mathrm{M})$ or GPS (50 and $400 \mu \mathrm{M})$ for $48 \mathrm{~h}$ after 4 days of differentiation, respectively, and observed by H\&E staining. (a) H\&E staining of cell morphology. Myotubes were examined with a phase-contrast light microscope (Olympus CKX41). Original magnification, $\times 200$. Scale bar indicates $100 \mu \mathrm{m}$. The arrow signs indicate the survival myotubes. (b) The number of myotubes was calculated as three random regions per group. Data are expressed as mean SD $(n=3)$. Different letters in lowercase indicate significance between one another as $p<0.05$. 


\subsection{Effect of GPS or GPSA on the FXT-Induced Muscle Atrophy-Associated Protein Expression and Signaling}

In the current study, we have found that FXT causes inhibition of $\mathrm{C} 2 \mathrm{C} 12$ muscle cell differentiation and a decrease in myotubular number, while GPS and GPSA improved such phenomena (Figures 1-3). Muscle atrophy is known to be associated with factors such as aging, inflammation, genetics, and drug side effects due to an imbalance between the anabolic and catabolic pathways that specify muscle mass $[20,21]$. Therefore, we further hypothesize that the possible mechanism of FXT-induced side effects of muscle atrophy is due to an imbalance between protein synthesis and degradation. Among the relevant molecular mechanisms resulting in muscle atrophy, the well-known one is muscle RING finger-1 (MuRF-1), which is closely associated with the loss of muscle mass [21]. MuRF1 is an E3 ubiquitin ligase expressed in skeletal muscle tissues and plays an important role in muscle remodeling. The upregulation of MuRF1 is involved in skeletal muscle atrophy [22]. The literature has demonstrated that different drugs attenuate muscle wasting by inhibiting MuRF-1 expression in animal and in vitro muscle wasting models [22,23].

In this study, the effect of GPS and GPSA on the expression of MuRF-1 protein in FXT-induced C2C12 muscle cells was investigated. As shown in Figure 4a, compared to the control group, FXT at $20 \mu \mathrm{M}$ significantly upregulated MuRF-1 protein expression in C2C12 muscle cells after $48 \mathrm{~h}(p<0.05)$, whereas the MuRF-1 protein was significantly downregulated $(p<0.05)$ after GPS and GPSA treatment (50 and $400 \mu \mathrm{M})$, with no statistical difference between doses. In other previous studies, it was also found that null mice in MuRF-1 reduced muscle atrophy caused by glucocorticoids, but the effect was not due to a reduction in protein degradation, but rather to the continued maintenance of protein synthesis [24]. Muscle differentiation (myogenesis) is associated with the activation of muscle-specific genes, in particular, genes encoding contractile proteins, such as $\mathrm{MyHC}$, are activated. Muscle atrophy may be associated with a shift in fiber type. Myosin heavy chain (MyHC) is a skeletal muscle-specific contractile protein expressed during muscle development [25]. The plant has been reported to contain several types of compounds that increase $\mathrm{MyHC}$ in $\mathrm{C} 2 \mathrm{C} 12$ cell lines [26]. Therefore, we also evaluated the effects of FXT on MyHC expression in C2C12 muscle cells and the regulatory effects of GPS and GPSA on MyHC. As shown in Figure 4b, compared to the control group, FXT at $20 \mu \mathrm{M}$ significantly downregulated MyHc protein expression in muscle cells after $48 \mathrm{~h}(p<0.05)$, whereas the MyHC protein was significantly upregulated after GPS and GPSA treatments (50 and $400 \mu \mathrm{M})(p<0.05)$, with no statistical difference between the two doses.

The role of signaling pathways associated with muscle hypertrophy was investigated in in vivo skeletal muscle hypertrophy and atrophy models based on the results of in vitro Akt/mTOR (mammalian target of rapamycin) studies [9,26-28]. The Akt/mTOR pathway is upregulated in hypertrophy and downregulated in muscle atrophy. The Akt protein kinase regulates protein metabolism and glucose transport, which are important for regulating cell growth and muscle fiber formation [29]. The amount of Akt expression, and more importantly the phosphorylation state representing Akt activation, increases throughout the muscle increase [26]. Figure $4 \mathrm{c}$ shows that FXT at $20 \mathrm{M}$ significantly inhibited the phosphorylated protein expression of Akt in C2C12 cells compared to control $(p<0.05)$, and both $50 \mu \mathrm{M}$ and $400 \mu \mathrm{M}$ GPS significantly restored the FXT-inhibited Akt protein phosphorylation $(p<0.05)$, while GPSA has no significant regulatory effect on Akt protein phosphorylation in FXT-induced C2C12 muscle cells. The overactivation of mammalian rapamycin (mTOR) signaling increases cell and tissue growth [30]. The AMP-activated protein kinase (AMPK) regulates mTOR by phosphorylating at Thr2446, thereby preventing AKT-mediated phosphorylation of mTOR at Ser2448. The AKT/mTOR pathway is an important regulator of skeletal muscle, downregulated in vivo during muscle atrophy and hypertrophy, and upregulated during hypertrophy [31]. As shown in Figure 4b, FXT significantly downregulated mTOR protein phosphorylation in C2C12 muscle cells compared to the control $(p<0.05)$, while only $400 \mu \mathrm{M}$ GPS significantly upregulated mTOR protein phosphorylation in FXT-treated C2C12 muscle cells $(p<0.05)$. 

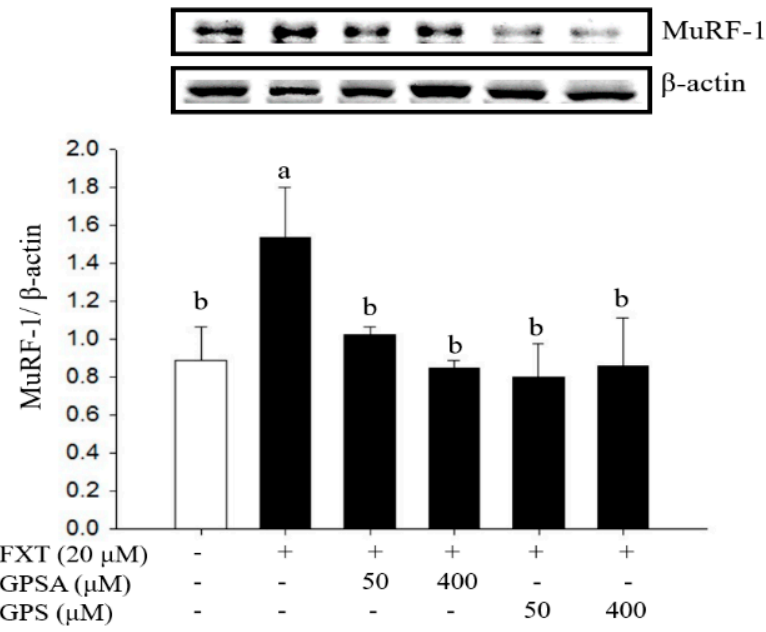

(a)

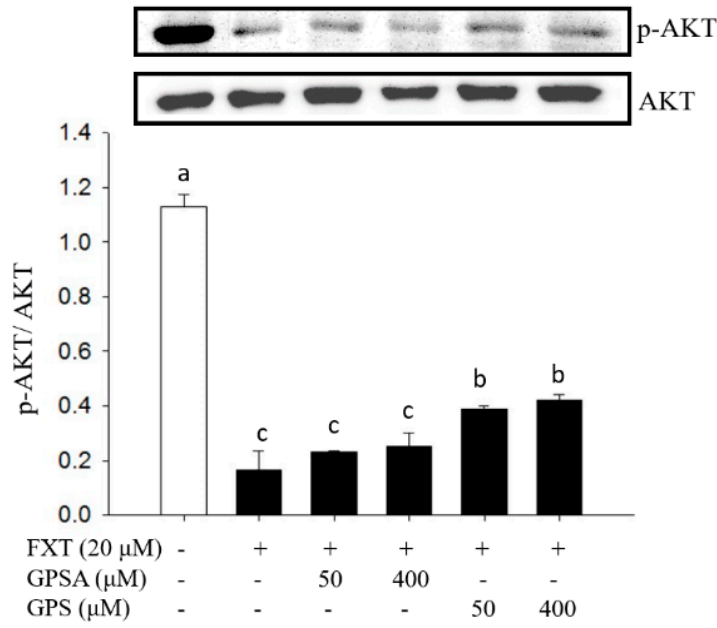

(c)
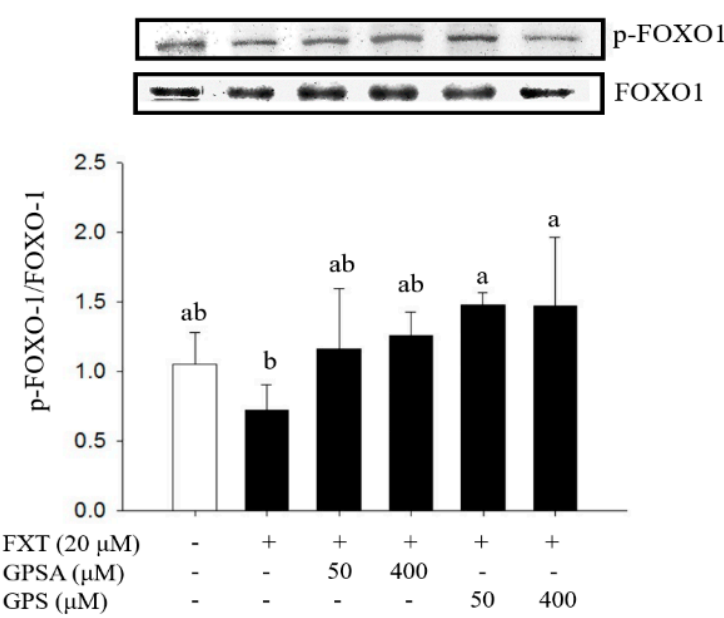

(e)

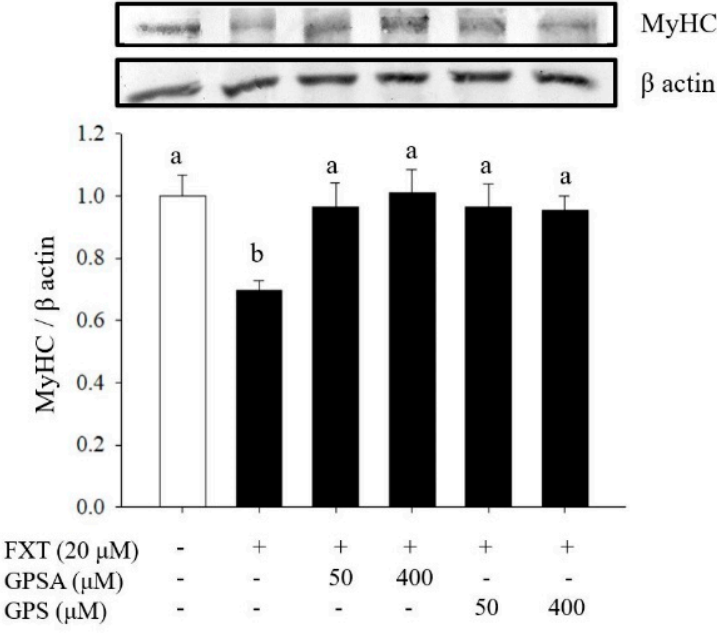

(b)

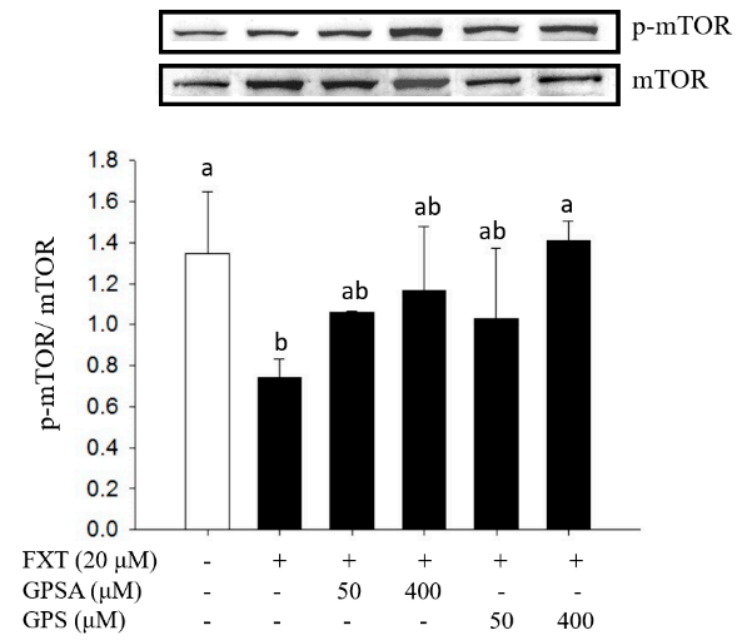

(d)

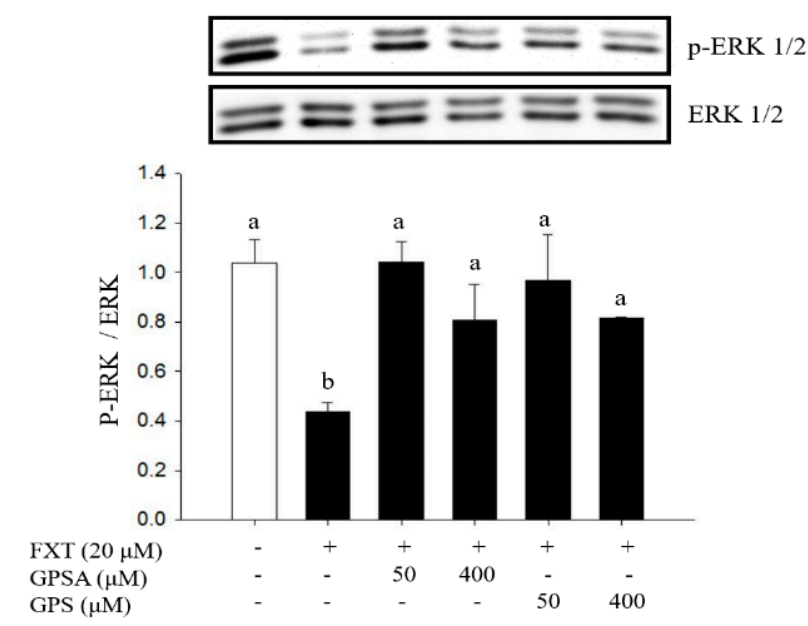

(f)

Figure 4. Effect of GPSA and GPS on the expression of muscle atrophy-related proteins in C2C12 cells induced by FXT. (a) MuRF-1, (b) MyHC, (c) AKT, (d) mTOR, (e) FOXO-1, and (f) ERK. C2C12 muscle cells were induced with FXT (20 $\mu$ M) and co-treated with GPSA or GPS at a dose of $50 \mathrm{M}$ and $400 \mathrm{M}$, respectively, for $48 \mathrm{~h}$. Data are expressed as mean \pm SD $(n=3)$. Different letters in lowercase indicate significance between one another as $p<0.05$. 
Forkhead proteins, especially FOXO1, play an important role in the regulation of whole-body energy metabolism. The distribution of FOXO1 in insulin-responsive tissues and its regulation through insulin-stimulated Akt phosphorylation enables FOXO1 to mediate a variety of important metabolic functions [32]. The skeletal muscle is the primary site of glucose processing in humans, accounting for $80-90 \%$ of insulin-stimulated glucose uptake. Therefore, maintaining its quality is very important for maintaining glucose homeostasis. Although the available data are controversial, there is evidence that FOXO1 plays a role in myocyte/myotube formation as well as myocyte size [33]. FOXO1 also promotes a reduction in muscle mass by regulating the expression of genes associated with muscle atrophy. It was noted that skeletal muscle atrophy is mediated through an Akt- and FOXO-1-dependent signaling pathway that promotes the expression of MuRF-1. In this study, the effects of GPS and GPSA on FOXO-1 expression in C2C12 muscle cells induced by FXT were investigated. The active form of FOXO- 1 consists of alanine residues replacing those serine/threonine residues phosphorylated by Akt, thus preventing their inactivation by phosphorylation. Figure 4e shows that FXT significantly downregulated FOXO-1 protein phosphorylation in $\mathrm{C} 2 \mathrm{C} 12$ muscle cells compared to the control $(p<0.05)$, whereas only GPS (50 and $400 \mathrm{M}$ ) significantly upregulated FOXO-1 protein phosphorylation in FXT-treated C2C12 muscle cells $(p<0.05)$.

Recently, specific signaling pathways have been implicated as the cause of muscle atrophy. Considering that proteins such as Murf- 1 and MyHC play a key role in mediating muscle atrophy, the attention was focused on these protein-dependent signals, especially those activated by mitogen-activated protein kinases (MAPKs). For example, an increase in p-ERK and a decrease in myosin heavy chain content can be observed in TNF $\alpha$-treated C2C12 myotubes [34], while a decrease in myosin and MyoD levels occurs in differentiated myoblasts exposed to cytokines [35]. Although MAPKs are involved in the pathogenesis of muscle atrophy, the role of the MAPKs is still largely debated. Presently, the role of ERK in the process of muscle atrophy in FXT-treated C2C12 muscle cells remains unknown. Thus, the effect of GPS and GPSA on the ERK1/2 protein phosphorylation in FXT-treated C2C12 muscle cells was also investigated in this study. As shown in Figure 4f, FXT significantly downregulated ERK1/2 protein phosphorylation in C2C12 muscle cells compared to the control $(p<0.05)$, whereas both GPS and GPSA (50 and $400 \mathrm{M})$ significantly upregulated ERK1 $/ 2$ protein phosphorylation in FXT-treated C2C12 muscle cells $(p<0.05)$. Our data here show that $\mathrm{p}$-ERK1/2 expression in C2C12 muscle cells decreases with FXT treatment and increases after GPS and GPSA treatment.

Moreover, FXT has been shown to induce pro-inflammatory phenomena in the liver by promoting TNF- $\alpha$ and triggers an increase in GPT and GOT, which in turn results in a decrease in hepatic catalase (CAT) and superoxide dismutase (SOD) to the point where the liver is affected by oxidative stress and results in apoptosis [36,37]. During such oxidative stress, FXT also affects lipid synthesis by promoting SREBP1c and decreases the action of the lipolytic-related hydroxylesterases CES1 and CES3 [38]. Thus, the effects of FXT on the metabolic hyperinflammatory response and oxidative stress are severe and widespread [39]. In previous studies on the mechanism of muscle atrophy, oxidative stress is an important factor in granulomatous dysfunction and causes muscle weakness, which may be strongly associated with FXT-induced muscle weakness. Recent studies have shown that oxidative stress induces Nrf2 gene expression and transcriptional activity in C2C12 skeletal muscle cells and rodent skeletal muscle [40-42]. The inhibition of Nrf2 using small interfering RNA (siRNA) enhanced myogenic differentiation of C2C12 cells [40]. Nrf2 may indeed be a key mechanism that needs to be clarified in FXT-mediated muscle atrophy, which will be investigated in future studies.

In summary, our study herein showed that the expression of proteins related to protein synthesis, such as MuRF-1 and MyHC, is reduced upon FXT administration. However, geniposide and/or geniposidic can attenuate the adverse effects of FXT by a possible mechanism that inhibits the activation of FOXO-1 transcription factors associated with impaired muscle protein synthesis by modulating the AKT/mTOR/ERK pathway. 


\section{Conclusions}

In this study, $\mathrm{C} 2 \mathrm{C} 12$ muscle cells were used to confirm the possible non-pharmacological therapeutic effects of GPS and GPSA on the muscle atrophy effects of FXT. Briefly, FXT downregulates the signaling pathway of p-ERK1/2, p-Akt, p-mTOR, and pFOXO in order to suppresses MyHC and promote MuRF-1 protein expression, resulting in enhanced protein degradation and cell muscle atrophy in C2C12 muscle cells. However, GPS and GPSA can regulate the atrophy mechanism caused by FXT in muscle cells, thus improving the imbalance of muscle synthesis, which has the potential to protect muscle cells from muscle loss caused by long-term FXT administration, suggesting that GPS and GPSA can be taken during medication to achieve the health benefits of maintaining muscle mass.

Author Contributions: S.-M.H.: Edited the paper. S.-Y.L. and M.-K.C.: Performed the experiment and collected the data. C.-C.P.: Proofread the manuscript. C.-L.H.: Designed the experiment and proofread the manuscript. All authors have read and agreed to the published version of the manuscript.

Funding: This research was funded by the Ministry of Science and Technology, Taiwan (MOST 02-2313-B-018-001-MY3 and MOST 107-2622-B-018-001-CC2).

Institutional Review Board Statement: Not applicable.

Informed Consent Statement: Not applicable.

Data Availability Statement: Not applicable.

Acknowledgments: This research work was supported by the Ministry of Science and Technology, Taiwan (MOST 02-2313-B-018-001-MY3 and MOST 107-2622-B-018-001-CC2).

Conflicts of Interest: The authors declare no conflict of interest.

\section{References}

1. Gao, Y.; Arfat, Y.; Wang, H.; Goswami, N. Muscle Atrophy Induced by Mechanical Unloading: Mechanisms and Potential Countermeasures. Front. Physiol. 2018, 9, 235. [CrossRef]

2. Lu, Y.; Ho, C.S.; Liu, X.; Chua, A.N.; Wang, W.; McIntyre, R.S.; Ho, R.C. Chronic administration of fluoxetine and pro-inflammatory cytokine change in a rat model of depression. PLoS ONE 2017, 12, e0186700. [CrossRef] [PubMed]

3. Visco, D.B.; Manhães-De-Castro, R.; Chaves, W.F.; Lacerda, D.C.; da Conceição Pereira, S.; Ferraz-Pereira, K.N.; Toscano, A.E. Selective serotonin reuptake inhibitors affect structure, function and metabolism of skeletal muscle: A systematic review. Pharmacol. Res. 2018, 136, 194-204. [CrossRef] [PubMed]

4. Bonaldo, P.; Sandri, M. Cellular and molecular mechanisms of muscle atrophy. Dis. Model. Mech. 2013, 6, 25-39. [CrossRef]

5. McKinnell, I.W.; Rudnicki, M.A. Molecular Mechanisms of Muscle Atrophy. Cell 2004, 119, 907-910. [CrossRef] [PubMed]

6. Sandri, M. Autophagy in health and disease. 3. Involvement of autophagy in muscle atrophy. Am. J. Physiol. Physiol. 2010, 298, C1291-C1297. [CrossRef]

7. Sandri, M. Autophagy in skeletal muscle. FEBS Lett. 2010, 584, 1411-1416. [CrossRef]

8. Milan, G.; Romanello, V.; Pescatore, F.; Armani, A.; Paik, J.-H.; Frasson, L.; Seydel, A.; Zhao, J.; Abraham, R.; Goldberg, A.L.; et al. Regulation of autophagy and the ubiquitin-proteasome system by the FoxO transcriptional network during muscle atrophy. Nat. Commun. 2015, 6, 6670. [CrossRef]

9. Sandri, M.; Barberi, L.; Bijlsma, A.Y.; Blaauw, B.; Dyar, K.; Milan, G.; Mammucari, C.; Meskers, C.; Pallafacchina, G.; Paoli, A.; et al. Signalling pathways regulating muscle mass in ageing skeletal muscle. The role of the IGF1-Akt-mTOR-FoxO pathway. Biogerontology 2013, 14, 303-323. [CrossRef]

10. Sanchez, A.M.; Candau, R.B.; Bernardi, H. FoxO transcription factors: Their roles in the maintenance of skeletal muscle homeostasis. Cell. Mol. Life Sci. 2013, 71, 1657-1671. [CrossRef]

11. Kim, S.; Koh, H. Role of FOXO transcription factors in crosstalk between mitochondria and the nucleus. J. Bioenerg. Biomembr. 2017, 16, 18224-18341. [CrossRef]

12. Gross, D.N.; Van den Heuvel, A.P.J.; Birnbaum, M.J. The role of FoxO in the regulation of metabolism. Oncogene 2008, 27, 2320-2336. [CrossRef]

13. Tan, S.; Wong, E. Mitophagy Transcriptome: Mechanistic Insights into Polyphenol-Mediated Mitophagy. Oxid. Med. Cell. Longev. 2017, 2017, 9028435. [CrossRef] [PubMed]

14. Webb, A.E.; Brunet, A. FOXO transcription factors: Key regulators of cellular quality control. Trends Biochem. Sci. 2014, 39, 159-169. [CrossRef] [PubMed]

15. Wang, X.; Zhang, F.; Xi, L.; Xia, W.; Wei, Y. Research Progress on Pharmacological Effects and Toxicity of Gardenia jasminoides Ellis and Related Mechanisms. Chin. J. Pharmacovigil. 2021, 18, 94. 
16. Li, N.; Li, L.; Wu, H.; Zhou, H. Antioxidative Property and Molecular Mechanisms Underlying Geniposide-Mediated Therapeutic Effects in Diabetes Mellitus and Cardiovascular Disease. Oxidative Med. Cell. Longev. 2019, 2019, 1-20. [CrossRef]

17. Zhang, X.; Liu, K.; Shi, M.; Xie, L.; Deng, M.; Chen, H.; Li, X. Therapeutic potential of catalpol and geniposide in Alzheimer's and Parkinson's diseases: A snapshot of their underlying mechanisms. Brain Res. Bull. 2021, 174, 281-295. [CrossRef]

18. Lautaoja, J.H.; Pekkala, S.; Pasternack, A.; Laitinen, M.; Ritvos, O.; Hulmi, J.J. Differentiation of Murine C2C12 Myoblasts Strongly Reduces the Effects of Myostatin on Intracellular Signaling. Biomolecules 2020, 10, 695. [CrossRef]

19. Kumar, P.; Nagarajan, A.; Uchil, P.D. Analysis of Cell Viability by the MTT Assay. Cold Spring Harb. Protoc. 2018, $2018,095505$. [CrossRef]

20. Michael, K. Relationship of skeletal muscle atrophy to functional status: A systematic research review. Biol. Res. Nurs. 2000, 2, 117-131. [CrossRef]

21. Rudrappa, S.S.; Wilkinson, D.J.; Greenhaff, P.L.; Smith, K.; Idris, I.; Atherton, P.J. Human Skeletal Muscle Disuse Atrophy: Effects on Muscle Protein Synthesis, Breakdown, and Insulin Resistance-A Qualitative Review. Front. Physiol. 2016, 7, 361. [CrossRef]

22. Ryu, Y.; Lee, D.; Jung, S.H.; Lee, K.-J.; Jin, H.; Kim, S.J.; Lee, H.M.; Kim, B.; Won, K.-J. Sabinene Prevents Skeletal Muscle Atrophy by Inhibiting the MAPK-MuRF-1 Pathway in Rats. Int. J. Mol. Sci. 2019, 20, 4955. [CrossRef]

23. Yuan, L.; Han, J.; Meng, Q.; Xi, Q.; Zhuang, Q.; Jiang, Y.; Han, Y.; Zhang, B.; Fang, J.; Wu, G. Muscle-specific E3 ubiquitin ligases are involved in muscle atrophy of cancer cachexia: An in vitro and in vivo study. Oncol. Rep. 2015, 33, 2261-2268. [CrossRef]

24. Baehr, L.M.; Furlow, J.D.; Bodine, S.C. Muscle sparing in muscle RING finger 1 null mice: Response to synthetic glucocorticoids. J. Physiol. 2011, 589, 4759-4776. [CrossRef]

25. Ferraro, E.; Pin, F.; Gorini, S.; Pontecorvo, L.; Ferri, A.; Mollace, V.; Costelli, P.; Rosano, G. Improvement of skeletal muscle performance in ageing by the metabolic modulator Trimetazidine. J. Cachexia Sarcopenia Muscle 2016, 7, 449-457. [CrossRef]

26. Yadav, A.; Singh, A.; Phogat, J.; Dahuja, A.; Dabur, R. Magnoflorine prevent the skeletal muscle atrophy via Akt/mTOR/FoxO signal pathway and increase slow-MyHC production in streptozotocin-induced diabetic rats. J. Ethnopharmacol. 2021, 267, 113510. [CrossRef]

27. Wang, M.; Ren, J.; Chen, X.; Liu, J.; Xu, X.; Li, X.; Zhao, D.; Sun, L. 20(S)-ginsenoside Rg3 promotes myoblast differentiation and protects against myotube atrophy via regulation of the Akt/mTOR/FoxO3 pathway. Biochem. Pharmacol. 2020, 180, 114145. [CrossRef]

28. Zheng, R.; Huang, S.; Zhu, J.; Lin, W.; Xu, H.; Zheng, X. Leucine attenuates muscle atrophy and autophagosome formation by activating PI3K/AKT/mTOR signaling pathway in rotator cuff tears. Cell Tissue Res. 2019, 378, 113-125. [CrossRef] [PubMed]

29. Son, Y.H.; Jang, E.J.; Kim, Y.W.; Lee, J.-H. Sulforaphane prevents dexamethasone-induced muscle atrophy via regulation of the Akt/Foxo1 axis in C2C12 myotubes. Biomed. Pharmacother. 2017, 95, 1486-1492. [CrossRef]

30. Kim, J.; Guan, K.-L. mTOR as a central hub of nutrient signalling and cell growth. Nat. Cell Biol. 2019, 21, 63-71. [CrossRef]

31. Sartori, R.; Romanello, V.; Sandri, M. Mechanisms of muscle atrophy and hypertrophy: Implications in health and disease. Nat. Commun. 2021, 12, 1-12. [CrossRef]

32. Menghini, R.; Casagrande, V.; Iuliani, G.; Rizza, S.; Mavilio, M.; Cardellini, M.; Federici, M. Metabolic aspects of cardiovascular diseases: Is FoxO1 a player or a target? Int. J. Biochem. Cell Biol. 2020, 118, 105659. [CrossRef]

33. Matsuda, R.; Uchitomi, R.; Oyabu, M.; Hatazawa, Y.; Kamei, Y. Metabolomic analysis of C2C12 myoblasts induced by the transcription factor FOXO 1. FEBS Lett. 2019, 593, 1303-1312. [CrossRef]

34. Penna, F.; Costamagna, D.; Fanzani, A.; Bonelli, G.; Baccino, F.M.; Costelli, P. Muscle Wasting and Impaired Myogenesis in Tumor Bearing Mice Are Prevented by ERK Inhibition. PLoS ONE 2010, 5, e13604. [CrossRef]

35. Swiderski, K.; Brock, C.J.; Trieu, J.; Chee, A.; Thakur, S.S.; Baum, D.M.; Gregorevic, P.; Murphy, K.T.; Lynch, G.S. Phosphorylation of ERK and dystrophin S3059 protects against inflammation-associated C2C12 myotube atrophy. Am. J. Physiol. Physiol. 2021, 320, C956-C965. [CrossRef]

36. Karimi-Khouzani, O.; Heidarian, E.; Amini, S.A. Anti-inflammatory and ameliorative effects of gallic acid on fluoxetine-induced oxidative stress and liver damage in rats. Pharmacol. Rep. 2017, 69, 830-835. [CrossRef]

37. Inkielewicz-Stepniak, I. Impact of fluoxetine on liver damage in rats. Pharmacol. Rep. 2011, 63, 441-447. [CrossRef]

38. Feng, X.-M.; Xiong, J.; Qin, H.; Liu, W.; Chen, R.-N.; Shang, W.; Ning, R.; Hu, G.; Yang, J. Fluoxetine Induces Hepatic Lipid Accumulation Via Both Promotion of the SREBP1c-Related Lipogenesis and Reduction of Lipolysis in Primary Mouse Hepatocytes. CNS Neurosci. Ther. 2012, 18, 974-980. [CrossRef] [PubMed]

39. Pieczenik, S.R.; Neustadt, J. Mitochondrial dysfunction and molecular pathways of disease. Exp. Mol. Pathol. 2007, 83, 84-92. [CrossRef]

40. Chung, L.-H.; Liu, S.-T.; Huang, S.-M.; Salter, D.M.; Lee, H.-S.; Hsu, Y.-J. High phosphate induces skeletal muscle atrophy and suppresses myogenic differentiation by increasing oxidative stress and activating Nrf2 signaling. Aging 2020, 12, 21446-21468. [CrossRef]

41. Kang, J.S.; Han, M.H.; Kim, G.-Y.; Kim, C.M.; Kim, B.W.; Hwang, H.J.; Choi, Y.H. Nrf2-Mediated HO-1 Induction Contributes to Antioxidant Capacity of a Schisandrae Fructus Ethanol Extract in C2C12 Myoblasts. Nutrients 2014, 6, 5667-5678. [CrossRef] [PubMed]

42. Whitman, S.A.; Long, M.; Wondrak, G.T.; Zheng, H.; Zhang, D.D. Nrf2 modulates contractile and metabolic properties of skeletal muscle in streptozotocin-induced diabetic atrophy. Exp. Cell Res. 2013, 319, 2673-2683. [CrossRef] [PubMed] 